\title{
A search for weakly interacting dark matter with the LUX experiment
}

\author{
C. Hall ${ }^{* a}{ }^{a}$ D. S. Akerib, ${ }^{b}$ X. Bai, ${ }^{c}$ S. Bedikian, ${ }^{d}$ A. Bernstein, ${ }^{e}$ A. Bolozdynya, ${ }^{f}$ \\ A. Bradley, ${ }^{b}$ S.B. Cahn, ${ }^{d}$ D. Carr, ${ }^{e}$ J. Chapman, ${ }^{g}$ K. Clark, ${ }^{b}$ T. Classen, ${ }^{h}$ A. Curioni, ${ }^{d}$ \\ E. Dahl, ${ }^{b}$ S. Dazeley, ${ }^{e}$ L. de Viveiros,${ }^{g}$ M. Dragowsky, ${ }^{b}$ E. Druszkiewicz, ${ }^{i}$ C. Faham, ${ }^{g}$ \\ S. Fiorucci, ${ }^{g}$ R. Gaitskell, ${ }^{g}$ M. Hanhardt, ${ }^{c}$ B. Holbrook, ${ }^{h}$ L. Kastens,${ }^{d}$ K. Kazkaz, ${ }^{e}$ \\ R. Lander, ${ }^{h}$ C. Lee, ${ }^{b}$ D.S. Leonard, ${ }^{a}$ K. Lesko, ${ }^{j}$ A. Lyashenko, ${ }^{d}$ D. Malling, ${ }^{g}$ \\ R. Mannino, ${ }^{k}$ D. McKinsey, ${ }^{d}$ D. Mei, ${ }^{l}$ J. Mock, ${ }^{h}$ M. Morii, ${ }^{m}$ J. Nikkel, ${ }^{d}$ P. Phelps, ${ }^{b}$ \\ U. Schroeder, ${ }^{i}$ T. Shutt, ${ }^{b}$ W. Skulski, ${ }^{i}$ P. Sorensen, ${ }^{e}$ J. Spaans, ${ }^{l}$ T. Stiegler, ${ }^{k}$ \\ R. Svoboda, ${ }^{h}$ M. Sweany, ${ }^{h}$ J. Thomson, ${ }^{h}$ J. Toke, ${ }^{i}$ M. Tripathi, ${ }^{h}$ J. Verbus,${ }^{g}$ N. Walsh, ${ }^{h}$ \\ R. Webb, ${ }^{k}$ J. White, ${ }^{k}$ F. Wolfs, ${ }^{i}$ M. Woods, ${ }^{h}$ and C. Zhang, ${ }^{l}$ \\ ${ }^{a}$ University of Maryland, Dept. of Physics, College Park MD 20742 \\ ${ }^{b}$ Case Western Reserve University, Dept. of Physics, 10900 Euclid Ave, Cleveland OH 44106 \\ ${ }^{c}$ South Dakota School of Mines and technology, 501 East St Joseph St., Rapid City SD 57701 \\ ${ }^{d}$ Yale University, Dept. of Physics, 217 Prospect St., New Haven CT 06511 \\ ${ }^{e}$ Lawrence Livermore National Laboratory, 7000 East Ave., Livermore CA 94551 \\ ${ }^{f}$ Moscow Engineering Physics Institute, 31 Kashirskoe shosse, Moscow 115409 \\ ${ }^{g}$ Brown University, Dept. of Physics, 182 Hope St., Providence RI 02912 \\ ${ }^{h}$ University of California Davis, Dept. of Physics, One Shields Ave., Davis CA 95616 \\ ${ }^{i}$ University of Rochester, Dept. of Physics and Astron., Rochester NY 14627 \\ ${ }^{j}$ Lawrence Berkeley National Laboratory, 1 Cyclotron Rd., Berkeley CA 94720 \\ ${ }^{k}$ Texas A \& M University, Dept. of Physics, College Station TX 77843 \\ ${ }^{l}$ University of South Dakota, Dept. of Physics, 414 E Clark St., Vermillion SD 57069 \\ ${ }^{m}$ Harvard University, Dept. of Physics, 17 Oxford St., Cambridge MA 02138
}

We review the design, status, and future plans for the LUX dark matter experiment, which aims to improve upon current dark matter limits by two orders of magnitude.

35th International Conference of High Energy Physics

July 22-28, 2010

Paris, France

* Speaker. 


\section{Introduction}

The existence and composition of the Milky Way's dark matter halo is one of the most fascinating questions in all of science. Cosmological and astrophysical evidence clearly indicates that the majority of the matter content of our universe consists of a new form which has yet to be observed in laboratories here on earth. One of the leading candidates for this new type of matter are the weakly interacting massive particles (WIMPs) which arise naturally in many extensions of the standard model of particle physics. Astrophysical WIMPs are expected to interact primarily with the atomic nuclei in the target material of a terrestrial detector, and therefore the signature of dark matter scattering is an excess of low energy nuclear recoil events which cannot be explained by conventional radioactivity.

The LUX collaboration was formed in 2007 with the goal of directly detecting WIMP dark matter with liquid xenon. The collaboration is composed of scientists with broad expertise in dark matter physics, neutrino physics, nuclear physics, and particle physics, including playing leading roles in the CDMS, CDMS-II, ZEPLIN-II, and XENON10 dark matter experiments. LUX is designed to achieve a WIMP scattering cross section sensitivity of $7 \times 10^{-46} \mathrm{~cm}^{2}$ for a WIMP mass of $100 \mathrm{GeV}$, about two orders of magnitude below current limits (see Figure 1).

Xenon is an attractive material for a dark matter search for several reasons. Its high mass $(\mathrm{A}=131.3)$ and high atomic number $(\mathrm{Z}=54)$ provides a favorable WIMP cross section while also giving good stopping power for environmental radioactivity. It does not have any long-lived isotopes which might contribute internal radioactive backgrounds, while trace contamination by the beta emitters ${ }^{85} \mathrm{Kr}$ and ${ }^{39} \mathrm{Ar}$ can be removed prior to operations via gas chromatography[1]. It can be continuously purified of other non-inert chemical and radioactive contaminants, even during detector operations. It can be contained within a single large vessel, which optimizes the surface area to volume ratio of the target material and minimizes exposure to radioactivity from the edges of the detector. Finally, by observing the quenching of the charge signal relative to the scintillation signal in nuclear recoil events, it allows backgrounds from beta and gamma interactions to be reduced by a factor of 180 while retaining $50 \%$ of the WIMP scattering signal.

\section{The LUX detector}

The LUX detector is a dual-phase TPC containing $350 \mathrm{~kg}$ of liquid xenon in an upright cylindrical vessel $(59 \mathrm{~cm}$ tall and $49 \mathrm{~cm}$ in diameter). Interactions in the liquid xenon bath are detected via the production of ionization charge and scintillation. The scintillation is collected by two arrays of photomultiplier tubes, one immersed in the liquid xenon at the bottom of the vessel, and one which observes the liquid from just above the liquid-gas interface. Ionization from the primary interaction is observed by drifting the charge vertically via an electric field to the liquid-gas interface. There the charge is extracted through the liquid surface into the gas phase, where secondary scintillation is generated as the charge is collected on an anode. The magnitude and location of the secondary scintillation measures the energy and $x-y$ coordinates of the ionization charge, while the drift time gives the $\mathrm{z}$ coordinate from the charge drift velocity. This allows the primary event to be localized to within one centimeter in all three dimensions. 
The LUX sensitivity goal requires an observational exposure of 30,000 kilogram-days. LUX will achieve this exposure after applying a fiducial cut which will reduce external backgrounds by four orders of magnitude through the powerful self-shielding effect of liquid xenon. The fiducial cut will leave $100 \mathrm{~kg}$ of useful xenon in the center of the detector, implying that an observation time of 300 days will be required. Beta and gamma background sources are required to contribute no more than 180 events in 300 days, so that only one background count is expected after the recoil discrimination cut. All LUX detector materials undergo careful screening to insure that their radioactivity level meets this goal. Special care has been taken with regard to the photomultiplier tubes, since these are expected to be the dominant source of gamma backgrounds. LUX employs a total of 122 Hamamatsu 2" PMTs (model R8778, QE = 30\%), and has worked closely with the vendor to monitor and reduce the activity of these devices. The PMTs delivered to LUX have a measured activity level of 10 and $2 \mathrm{mBq} / \mathrm{PMT}$ for ${ }^{238} \mathrm{U}$ and ${ }^{232} \mathrm{Th}$, respectively, improving upon the LUX goals by factor of three. Considerable effort was also dedicated to identifying an acceptable material for the LUX cryostat. Previous experiments have relied upon materials which suffer either from marginal radioactivity (stainless steel) or poor mechanical properties (copper). The LUX cryostat has been constructed out of clean titanium, with activity levels of $<0.4 \mathrm{mBq} / \mathrm{kg}$, again improving on LUX's background goals while meeting regulatory requirements for a pressure vessel. All other components of the LUX internal detector are required to contribute less than $10 \%$ to the expected background rate.

LUX has been designed to address and resolve numerous issues which will inevitably be faced by future experiments at the ton-scale and beyond. Among these features are:

- Water shield. The LUX detector will be immersed in a 8 meter diameter, 6 meter tall, 300 ton water shield to reduce environmental radioactivity due to gammas and neutrons to a negligible level. The water shield is instrumented with PMTs to allow it to be employed as a cosmic ray veto via detection of Cerenkov radiation.

- Xenon purity. The LUX xenon handling system is designed to repurify the entire xenon target material once per day, allowing electronegative impurities such as oxygen and water to be rapidly removed during detector commissioning. A key component of this system is a custom xenon heat exchanger, designed and tested by LUX, which allows xenon to be evaporated and re-condensed with a power efficiency of $96 \%$, considerably reducing the amount of cooling power required by the experiment.

- Cryogenics. LUX employs a unique thermosyphon system to cool the titanium cryostat [3]. The thermosyphons deliver more than $1000 \mathrm{~W}$ of cooling power (far more than needed) to the LUX cryostat through the water shield.

- Beta calibration sources. LUX will deploy two internal calibration sources based on ${ }^{83 m} \mathrm{Kr}$ [2] and tritium to determine the energy scale and nuclear recoil discrimination factor of the detector. This resolves the self-shielding issue which will affect any external gamma calibration source. 

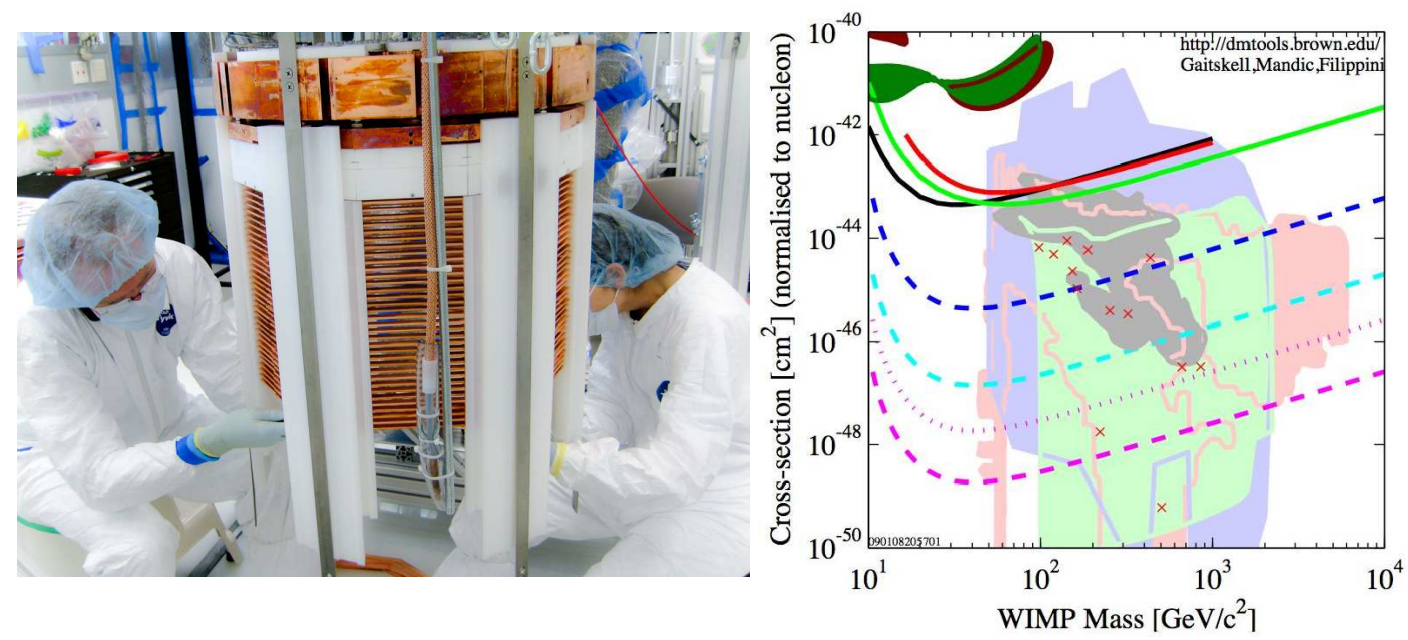

Figure 1: Left: The LUX detector, under assembly at the Sanford Lab in Lead, South Dakota, in the summer of 2010. Right: the WIMP cross-section sensitivity of LUX (dark blue dashed line), LZS (cyan dashed line), and LZD (purple dashed line).

\section{Status of LUX}

The LUX detector has been assembled with final detector components in a class 1000 clean room facility on the surface at the Sanford Lab in Lead, South Dakota. The detector will be commissioned in the winter of 2011 by deploying it into a test water shield which has been constructed in the surface lab. The primary goal of the surface run is to demonstrate good xenon purity. Meanwhile, 4850 feet below the surface laboratory, the cavern where Ray Davis performed the historic Homestake solar neutrino experiment is being outfitted with the infrastructure required to host the LUX experiment for physics data taking. The detector is designed to be fully transportable from the surface to the underground lab without breaking its vacuum seal, which will allow detector operations to resume quickly once the space is prepared. This will occur in the fall of 2011.

\section{Beyond LUX}

The LUX and ZEPLIN-III collaborations have agreed to jointly execute future liquid xenon dark matter experiments. The new collaboration, known as LZ, is currently developing proposals to rapidly replace LUX with a 1.5 ton detector in the same underground location (the LZS experiment), and also to construct a 20 ton experiment for the 7400 foot level of the proposed DUSEL facility (the LZD experiment). LZS and LZD will improve upon the LUX sensitivity by factors of 50 and 700, respectively (see Figure 1). The LZ groups will also jointly participate in LUX operations and data analysis, fully uniting the two collaborations in the near future.

\section{References}

[1] A. I. Bolozdynya, et al., Nucl. Instr. Meth. A 57950 (2007).

[2] L.W.Kastens, et al., Journ. Instrum. 5 P05006 (2010).

[3] A. Bolozdynya, et. al., IEEE Trans. Nucl. Sci., Vol. 562309 (2009). 